\title{
Twelve-year analysis of microbial keratitis trends at a UK tertiary hospital
}

SZ Tan ${ }^{1,2}$, A Walkden ${ }^{1,2}, \mathrm{~L} \mathrm{Au^{1,2 } , \text { C Fullwood }}{ }^{3,4}$, A Hamilton ${ }^{1}$, A Qamruddin ${ }^{5}$, M Armstrong ${ }^{5}$, AK Brahma ${ }^{1,2}$ and F Carley ${ }^{1,2}$

\begin{abstract}
Purpose To investigate the frequencies, trends, and in vitro drug susceptibilities of the causative pathogens in microbial keratitis in Manchester Royal Eye Hospital. Patients and methods Corneal scrape results recorded by the microbiology service between 2004 and 2015 were extracted from an established database. A total of 4229 corneal scrape specimens were identified from an established database. First-line antibiotic treatment in our centre during the study period was ofloxacin and second line was cefuroxime and gentamicin.
\end{abstract}

Results Mean age was $45.9 \pm 21.0$. A total of 1379 samples $(32.6 \%)$ were culture positive.

One hundred forty-eight (10.7\%) specimens cultured multiple organisms. Of the 1539 organisms identified, $63.3 \%$ were

Gram-positive bacteria, 27.3\% Gram-negative bacteria, $7.1 \%$ fungi, and $2.3 \%$

Acanthamoebae. A decreasing trend in Grampositive isolates was found together with a stable trend in Gram negatives and an increasing trend in Acanthamoeba and fungi. There appeared to be a significant increasing trend of Moraxella infection $(P=0.001)$.

In all, 83.1 and $\mathbf{9 0 . 8 \%}$ of Gram-positive and -negative isolates tested were susceptible to ofloxacin, respectively. Cefuroxime covered $86.6 \%$ of Gram-positive and $61.4 \%$ of Gram-negative isolates, whereas gentamicin covered 88.8 and $96.5 \%$ of Gram-positive and -negative isolates, respectively.

Conclusion We found a change in the type of Gram-negative organisms isolated over time, with the Moraxella species on the rise. Reassuringly, no significant increase in resistance was observed in vitro for any of the commonly used antibiotics. Ofloxacin remains a good first-line antibiotic treatment but duo-therapy does have broader coverage and should be considered in nonresponsive cases.
Eye (2017) 31, 1229-1236; doi:10.1038/eye.2017.55; published online 28 April 2017

\section{Introduction}

Microbial keratitis remains a serious cause of corneal opacification and sight loss worldwide. ${ }^{1}$ Timely diagnosis and appropriate use of topical antimicrobial therapy are critical to effective management. Empirical use of broad-spectrum antimicrobial therapies is often the primary treatment modality, which is then tailored according to clinical response, cultured organisms, and their sensitivities. Increasing antimicrobial resistance due to inappropriate use of antibiotics has become a major public health issue. The Department of Health, UK, has recently published an integrated '5-year antimicrobial resistance strategy' to tackle this issue. ${ }^{2}$ The overarching strategy is to encourage the optimal use of antimicrobial agent for each clinical scenario. Geographical variation in microbial spectrum and therapy is well researched. It is therefore imperative that local microbial trends are analysed to enable effective, evidence-based treatment algorithms to be used during the initial stages of clinical management.

The aim of this study was to determine and evaluate the frequencies, trends, and drug susceptibilities of the causative pathogens resulting in microbial keratitis over a 12-year period at the Manchester Royal Eye Hospital, a tertiary referral centre in the UK.

\section{Materials and methods}

This study was conducted in accordance with the Declaration of Helsinki and approval was obtained from the National Integrated Research Application System.

All corneal scrape specimens received and examined by the microbiology service between 1 January 2004 and 31 December 2015 were
${ }^{1}$ Centre for Ophthalmology and Vision Sciences, Faculty of Medical and Human Sciences, University of Manchester, Manchester, UK

${ }^{2}$ Manchester Royal Eye Hospital, Central Manchester University Hospitals NHS Foundation Trust, Manchester Academic Health Science Centre, Manchester, UK

${ }^{3}$ Centre for Biostatistics, Division of Population Health, Health Services Research and Primary Care, School of Health Sciences, Faculty of Biology, Medicine and Health, University of Manchester, Manchester, UK

${ }^{4}$ Research and Innovation, Central Manchester University Hospitals NHS Foundation Trust, Manchester, UK

${ }^{5}$ Microbiology Department, Clinical Sciences Building, Manchester Royal Infirmary, Manchester, UK

Correspondence:

SZ Tan, Ophthalmology, Manchester Royal Eye Hospital, Central Manchester University Hospitals NHS Foundation Trust, Manchester Academic Health Science Centre, Manchester M13 9WL, UK Tel: +44 (0)16 1276 1234; Fax: +44 (0)1617010242. E-mail: shizhuan@gmail.com

Received: 4 December 2016 Accepted in revised form: 12 February 2017 Published online: 28 April 2017 
retrieved from an established electronic database.

Data collected including age of patient, date of scrape, Gram film, isolates, antimicrobial susceptibilities were organised onto a pre-designed pro forma.

A standard corneal scrape kit consisted of a glass slide for Gram stain, of which results were available within $6 \mathrm{~h}$ from the laboratory, two blood agar plates and one chocolate agar plate $\left(6 \% \mathrm{CO}_{2}\right.$, at $\left.37^{\circ} \mathrm{C}\right)$. Sabouraud's agar plate (air at $30^{\circ} \mathrm{C}$ ) was used for fungus and a non-nutrient agar seeded with Escherichia coli for Acanthamoeba isolation, when there was high clinical suspicion for these infections. The blood and chocolate agar plates were incubated for 5 days and the non-nutrient agar was incubated for 7 days.

Corneal material was routinely obtained using a sterile $23 \mathrm{G}$ needle and inoculated in a ' $\mathrm{C}$ ' shape shallowly onto the culture plates. Any isolates seen growing on the ' $C$ ' inoculum were considered significant. Identification of organisms was achieved through the Biochemical methods (Vitek2, Biomerieux, Basingstoke, UK) or by mass spectrometry (MALDI-TOF, Bruker, Coventry, UK). The technique for isolation had remained consistent throughout the study period.

Significant isolates were tested against selected antibiotics in accordance with local protocol using both the British Society of Antimicrobial Chemotherapy methodology (www.BSAC.org.uk) and the Vitek 2XL system (www.biomerieux.co.uk). Based on the minimum inhibitory concentration systemic break points, all isolates were classified as susceptible or resistant. For staphylococci, antimicrobial susceptibility testing was achieved through the use of AST P635 cards on the Vitek2 system. Oxacillin and cefoxitin were tested to infer MecA production. Isolates that tested resistant to cefoxitin were reported as methicillin-resistant staph. aureus.

Cephalosporins of any class were not used to infer production of MecA within staphylococci. Beta lactamase production by enterobacteriaceae was inferred from resistance to third and fourth generation cephalosporins tested within the Vitek N206 AST cards. Cefuroxime was only used as a screening agent for beta lactamase production within Neisseria gonorrhoea. Candida species susceptibility testing was performed using Vitek YSO7 cards. Other fungal isolates, such as aspergillus and Fusarium, were referred to the regional mycology reference unit at the University of South Manchester, where a minimum inhibitory concentration method was performed for all anti-fungal agents.

Statistical analysis was performed using Microsoft Excel, Xlstat 2016.1, and R v3.2.4. To examine trends in the data, the study period was divided into four groups of 3-year intervals: 2004-2006, 2007-2009, 2010-2012, and 2013-2015. Graphical representation was used to gain an understanding of the structure of the data, while the
Cochran-Armitage trend test was used to detect trends. A $P$-value of $<0.05$ was considered statistically significant, with consideration made for multiple testing in the interpretation.

\section{Results}

\section{Overall trend analysis}

A total of 4229 corneal scrape specimens were analysed during the study period. Mean patient age was $45.9 \pm 21.0$ (range 0.0-97.4). Of these, 1379 samples (32.6\%) were culture positive and $148(10.7 \%)$ specimens cultured multiple organisms (range 1-4). There was a peak in the percentage of positive culture in 2007 of $48.8 \%$, with a downward trend towards the end of the study period, from $34.9 \%$ positive culture rate in 2004 to $23.2 \%$ in 2015 .

A total of 1539 organisms were identified, of which 63.3 and $27.3 \%$ were Gram-positive and Gram-negative bacteria, respectively, $7.1 \%$ were fungi, and $2.3 \%$ were Acanthamoebae.

There was a suggestion of a decreasing trend in the percentage of Gram-positive bacteria isolated, a stable trend for Gram-negative bacteria, and an increasing trend for fungi and Acanthamoeba (Figure 1). When the study period was divided into four groups of 3-year intervals, trend analysis showed statistical significance for Grampositive bacteria $(P<0.001)$, fungi $(P=0.005)$, and Acanthamoeba $(P<0.001)$ but not for Gram-negative bacteria $(P=0.3$; Table 1$)$.

The most commonly isolated organism was coagulase negative staphylococcus (CNS), which accounted for $38.5 \%$ of all Gram-positive bacteria, although there appeared to be a decreasing trend over 12 years $(P<0.001)$. In contrast, Staphylococcus aureus (SA) and

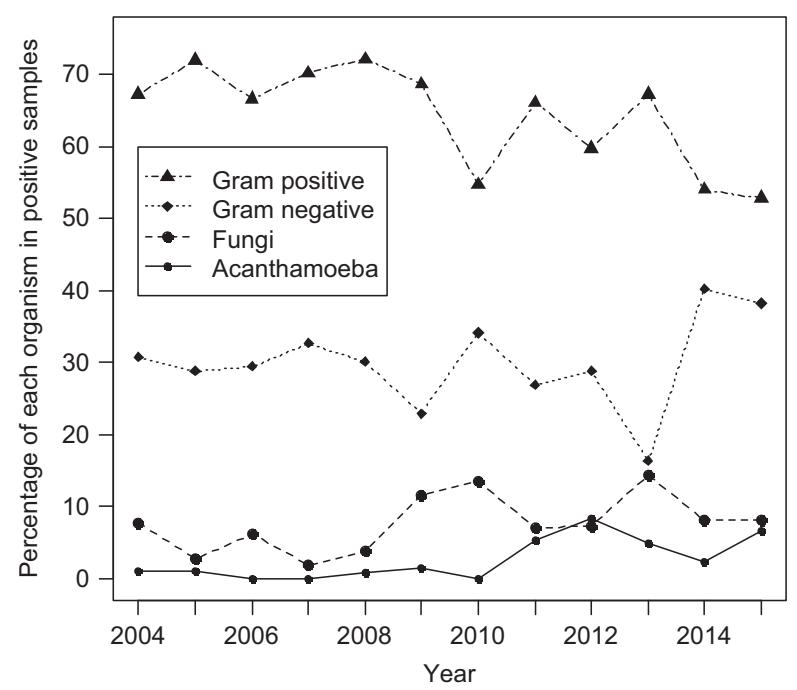

Figure 1 The trend of identified organisms over 12 years. 
Table 1 Trends in different organisms over 12 years study period

\begin{tabular}{|c|c|c|c|c|c|c|}
\hline Organisms & $\begin{array}{c}2004-2006 \\
\text { N (\%) }\end{array}$ & $\begin{array}{c}2007-2009 \\
\text { N (\%) }\end{array}$ & $\begin{array}{c}\text { 2010-2012 } \\
\text { N (\%) }\end{array}$ & $\begin{array}{c}2013-2015 \\
\text { N (\%) }\end{array}$ & $\begin{array}{c}\text { Total } \\
\text { N (\%) }\end{array}$ & P-value \\
\hline Gram positive $\downarrow$ & $263(67.8)$ & $287(68.3)$ & $226(59.0)$ & 198 (57.1) & 974 (63.3) & $<0.001$ \\
\hline Streptococcus $\uparrow$ & $46(17.5)$ & $51(17.8)$ & $60(26.5)$ & $48(24.2)$ & 205 (21.1) & 0.010 \\
\hline Staph. aureus $\uparrow$ & $53(20.2)$ & $63(22.0)$ & $52(23.0)$ & $65(32.8)$ & $233(23.9)$ & 0.003 \\
\hline CNS $\downarrow$ & $128(48.7)$ & $121(42.2)$ & $80(35.4)$ & $46(23.2)$ & 375 (38.5) & $<0.001$ \\
\hline Bacillus $\downarrow$ & $16(6.1)$ & $22(7.7)$ & $4(1.8)$ & $5(2.5)$ & $47(4.8)$ & 0.008 \\
\hline Others* $\uparrow$ & $20(7.6)$ & $30(10.5)$ & 30 (13.3) & $34(17.2)$ & 114 (11.7) & 0.001 \\
\hline Gram negative & $103(26.5)$ & $108(25.7)$ & $108(28.2)$ & $101(29.1)$ & $420(27.3)$ & 0.300 \\
\hline Pseudomonas & $46(44.1)$ & 37 (33.9) & $43(38.4)$ & $30(30.4)$ & $156(37.1)$ & 0.070 \\
\hline Moraxella $\uparrow$ & $16(15.7)$ & $22(20.1)$ & $16(13.8)$ & $39(34.9)$ & $93(22.1)$ & 0.001 \\
\hline Serratia & 13 (12.6) & $9(8.3)$ & $12(11.1)$ & $10(9.9)$ & 44 (10.5) & 0.700 \\
\hline Others $^{\phi}$ & $28(27.2)$ & $40(37.0)$ & 37 (34.3) & $22(21.8)$ & $127(30.2)$ & 0.400 \\
\hline Fungi $\uparrow$ & $20(5.2)$ & $22(5.2)$ & $34(8.9)$ & $33(9.5)$ & 109 (7.1) & 0.005 \\
\hline Acanthamoeba $\uparrow$ & $3(0.8)$ & $3(0.7)$ & $15(3.9)$ & $15(4.3)$ & $36(2.3)$ & $<0.001$ \\
\hline
\end{tabular}

Abbreviation: CNS, coagulase negative Staphylococcus.

$\downarrow$ Indicates a significant decreasing trend; $\uparrow$ indicates a significant increasing trend.

Others $^{*}=$ other Staphylococcus including Staph. capitis, Staph. warneri, Staph. hominis, Staph. saprophyticus, Abiotrophia, Clostridium, diphtheroids, Finegoldia magna, Gemella species, Nocardia transvalensis, Enterococcus, Micrococcus, Propionibacterium.

Others $^{\phi}=$ Achromobacter, Acinetobacter, Coliform, Enterobacter, E. coli, H. influenza, Klebsiella, sphingomonas, Stenotrophomonas, Brevundimonas diminuta, Chryseobacterium indologenes, Citrobacter, Elizabethkingia miricola, Morganella morganii, Neisseria, Pantoea, Proteus, Rhizobium radiobacter.

Bold values indicate $P<0.005$.

Streptococcus species appeared to be on the rise $(P=0.003$, $P=0.01$, respectively; Table 1$)$. A total of 15 cases of methicillin-resistant staph. aureus were isolated over the study period.

Pseudomonas species (with $86.0 \%$ being Pseudomonas aeruginosa) was found to be the most commonly isolated Gram-negative organism. Although numbers appeared to be decreasing over the study period, this was nonsignificant on testing for trend $(P=0.07)$. However, a significant increase in trend for the Moraxella species $(P=0.001)$ was evident (Table 1$)$.

Among the fungal species, yeast and Fusarium were the two commonest isolates, which when combined accounted for $78.9 \%$ of all fungal isolates (53.2 and $25.7 \%$, respectively).

\section{Antimicrobial susceptibility}

Gram positive The antibiotic susceptibility rate for Grampositive organisms is demonstrated in Table 2. There was no observed increase in resistance for any of the antibiotics tested (fusidic acid, vancomycin, chloramphenicol, ofloxacin and gentamicin) but an increase in susceptibility was observed for ofloxacin.

It was found that $88.5 \%$ of CNS were susceptible to chloramphenicol, in contrast to $95.6 \%$ of SA and $99.4 \%$ of Streptococcus species. Gentamicin was found to be an effective choice of antibiotics for CNS (97.3\% susceptibility) and SA (99.6\% susceptibility) but all the Streptococcus species tested were resistant to gentamicin. Susceptibility to ofloxacin was the highest for SA (91.2\%), followed by CNS (86.2\%) and Streptococcus species
(59.3\%) although there appeared to be an increasing susceptibility among the Streptococcus species. Both SA and Streptococcus species were found to have high susceptibility to cefuroxime (100.0 and $96.8 \%$ ) and less so for CNS $(63.2 \%)$ but the susceptibility test was performed for only a very small number of samples in the second half of the study period (Table 3). All the methicillinresistant staph. aureus cases were susceptible to vancomycin and ofloxacin.

Gram negative The antibiotic susceptibility rate for Gram-negative organisms is demonstrated in Table 2. Overall, there was an increasing trend of susceptibility towards chloramphenicol $(P<0.001)$ and cefuroxime $(P<0.001)$ among the Gram-negative organisms.

Moraxella species were highly susceptible to chloramphenicol $(100 \%)$, cefuroxime $(100 \%)$, and ciprofloxacin $(99.2 \%)$ and gentamicin $(98.8 \%)$ but only $87.9 \%$ were susceptible to ofloxacin. Pseudomonas species remained highly susceptible to ciprofloxacin (100\%), gentamicin $(100 \%)$, tobramycin $(100 \%)$, ceftazidime (98.7\%), and ofloxacin (95.0\%; Table 4$)$.

Fungi In all, $94.6 \%$ of fungal species were sensitive to amphotericin, $95.7 \%$ to voriconazole, and $97.4 \%$ to flucytosine. All Fusarium and yeast species tested for voriconazole were susceptible to it; two out of the 12 Fusarium species tested for amphotericin susceptibility displayed resistance while there was no resistance observed among the yeast species for amphotericin. 
Table 2 Antibiotic susceptibility in both Gram-positive and Gram-negative organisms

\begin{tabular}{|c|c|c|c|c|c|c|}
\hline & $\begin{array}{c}2004-2006 \\
\mathrm{~N}(\%)\end{array}$ & $\begin{array}{c}2007-2009 \\
\text { N (\%) }\end{array}$ & $\begin{array}{c}2010-2012 \\
\text { N (\%) }\end{array}$ & $\begin{array}{c}2013-2015 \\
\text { N (\%) }\end{array}$ & $\begin{array}{l}\text { Total } \\
\text { N }(\%)\end{array}$ & P-value \\
\hline Gram positive & 263 & 287 & 226 & 198 & 974 & \\
\hline Chloramphenicol & $201(89.3)$ & $197(92.5)$ & $144(94.7)$ & $137(93.2)$ & $679(92.1)$ & 0.10 \\
\hline Ofloxacin & $156(76.8)$ & $97(75.2)$ & $100(87.7)$ & $103(92.8)$ & $456(83.1)$ & $<0.001$ \\
\hline Fusidic acid & $123(64.4)$ & $105(67.3)$ & $66(68.8)$ & $73(67.6)$ & $367(67.0)$ & 0.50 \\
\hline Vancomycin & $113(100.0)$ & $141(100.0)$ & 138 (99.3) & $140(100.0)$ & $532(99.8)$ & 0.70 \\
\hline Gentamicin & $188(87.4)$ & 176 (87.6) & 107 (87.0) & 108 (93.1) & $579(88.8)$ & 0.21 \\
\hline Cefuroxime & $139(88.5)$ & $104(81.3)$ & $38(92.7)$ & $22(91.2)$ & $303(86.6)$ & 0.13 \\
\hline Gram negative & 103 & 108 & 108 & 101 & 420 & \\
\hline Amikacin & $19(100.0)$ & $30(93.8)$ & $64(100.0)$ & $46(97.9)$ & $159(97.9)$ & 0.89 \\
\hline Ceftazidime & 54 (91.5) & $48(88.9)$ & $67(95.7)$ & 47 (92.2) & $216(92.1)$ & 0.55 \\
\hline Chloramphenicol & $32(56.1)$ & $43(63.2)$ & $29(87.9)$ & 61 (95.3) & $165(75.6)$ & $<0.001$ \\
\hline Ciprofloxacin & $60(98.4)$ & $56(100.0)$ & $75(98.7)$ & $79(98.8)$ & $270(98.9)$ & 0.80 \\
\hline Ofloxacin & 77 (89.5) & $61(84.7)$ & $46(93.9)$ & $58(95.1)$ & $242(90.8)$ & 0.14 \\
\hline Gentamicin & $94(97.9)$ & $92(93.9)$ & $80(94.1)$ & $58(100.0)$ & $324(96.5)$ & 0.78 \\
\hline Cefuroxime & $27(48.2)$ & 33 (49.3) & $28(68.3)$ & $47(79.7)$ & $135(61.4)$ & $<0.001$ \\
\hline
\end{tabular}

Bold values indicate $P<0.005$.

\section{Discussion}

To the best of our knowledge, this is the largest and the longest data series used to analyse the current trends of microbial keratitis in the UK. The overall yield of positive cultures was $32.6 \%$ in our study, in contrast to $54.0 \%$ in the Oxford study, ${ }^{3} 49.3 \%$ in the Taiwanese study ${ }^{4}$ and $38.4 \%$ in the Portuguese study. ${ }^{5}$ Interestingly, we found that the percentage of positive cultures declined over the study period. This could partly be explained by the frequent use of antibiotics by general practitioners or chemists in the primary care before patients presented to our eye unit. The other explanations could be that insufficient samples were obtained from the cornea or that the scraping technique was inadequate. Corneal scrapes may have also been performed more frequently than required in the recent years on non-infective cases such as marginal keratitis.

Our data showed Gram-positive organisms to be the most common isolate although there appeared to be an overall decreasing trend of these microbes being isolated over time. There was also a shifting trend towards increasing numbers of fungi and Acanthamoeba species isolated. Gram-negative species overall remained similar, although in latter years the levels of variability appeared to be increasing. Gram-positive organisms were also found to be the commonest isolates in the Oxford series by Orlans et $a l^{3}$ but the authors did not find a trend shift during the period 1999-2009. In our study, CNS was the commonest isolate but we observed a reducing trend in this organism with a shift towards an increasing trend of SA and Streptococcal isolates. CNS was also found to be the commonest isolate in other UK series, ${ }^{3,6}$ as well as the Indian ${ }^{7}$ and the Saudi Arabian ${ }^{8}$ series. Conversely, an increasing trend of CNS was observed by Orlans et al. ${ }^{3}$
When compared to other regions in the world, SA was found to be the commonest isolate in Portugal, ${ }^{5}$ whereas Pseudomonas species were the commonest isolates in Taiwan, ${ }^{4}$ China, ${ }^{9}$ and South Florida. ${ }^{10}$

First-line treatment in our centre is the secondgeneration fluoroquinolone, ofloxacin. Our study showed that $83.1 \%$ of Gram-positive organisms and $90.8 \%$ of Gram-negative organisms were susceptible to this antibiotic. However, it is important to note that antibiotics given topically and intensively may achieve a high enough concentration to have a bactericidal effect on the microorganism and hence, the clinical susceptibility rate for ofloxacin may well be higher than the rate reported in vitro. Although results of in vitro microbial testing have been shown to be predictive of therapeutic response in bacterial keratitis, ${ }^{11}$ laboratory results should only be used as a guide to continue or discontinue treatment in context with the clinical response to treatment. The alternative first-line treatment in the UK is duo-therapy, usually with cefuroxime and gentamicin. Our study showed that cefuroxime had a 86.6 and $61.4 \%$ susceptibility rate to Gram-positive and Gram-negative organisms, respectively, and gentamicin had a 88.8 and $96.5 \%$ susceptibility rate to the two groups, respectively, which when used in combination did appear to increase the overall coverage of organisms compared to ofloxacin alone. However, gentamicin is toxic to the ocular surface and duo-therapy is more difficult to administer. Hence, newer-generation fluoroquinolone as monotherapy is often preferred to duo-therapy due to its high tolerability. The use of monotherapy is also supported by a randomised controlled trial, which showed similar efficacy of fluoroquinolone compared to duo-therapy therapy (cefazolin and tobramycin) with fewer side effects. ${ }^{12}$ However, monotherapy with fluoroquinolone 

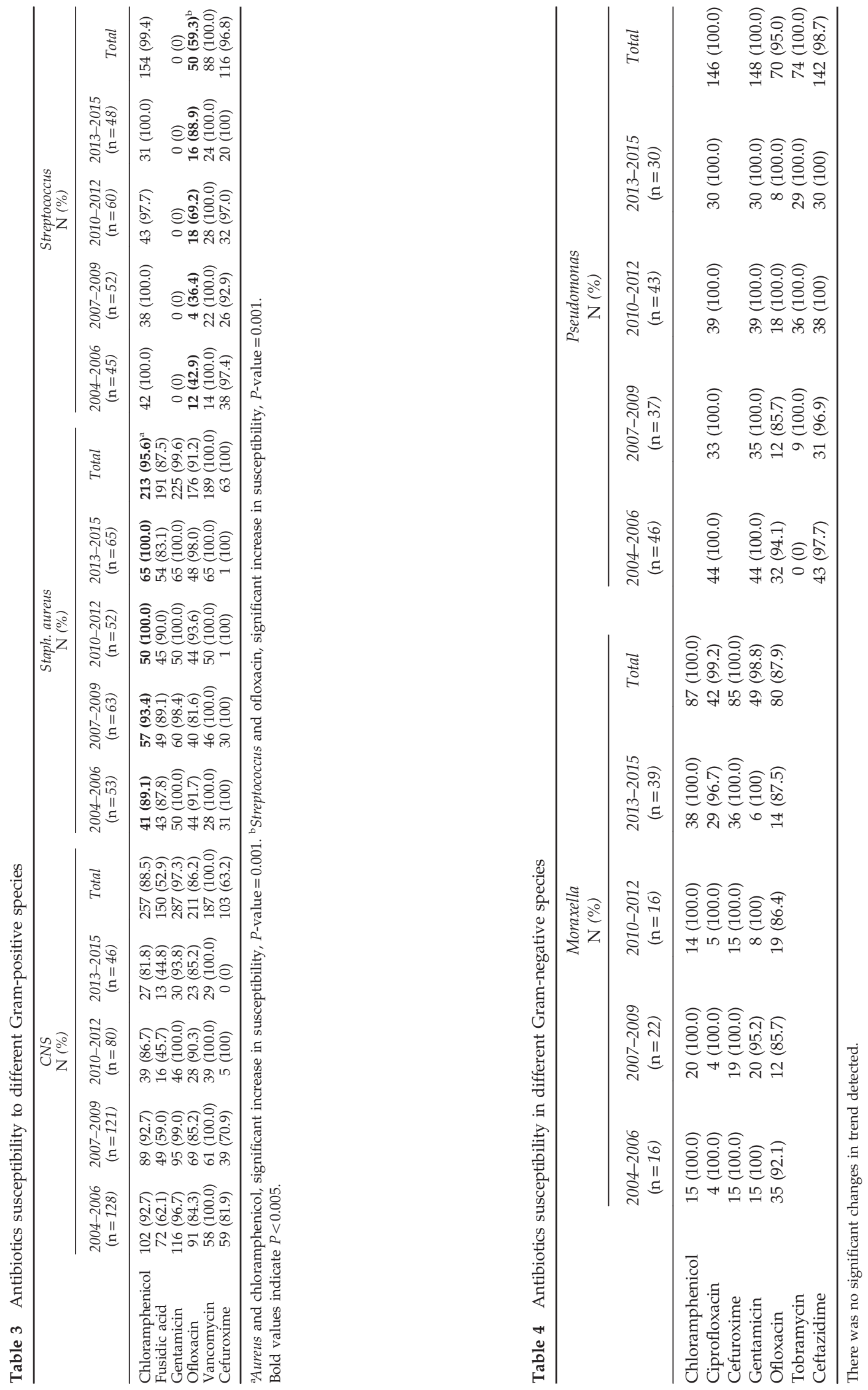
has its pitfall. Goldstein et al ${ }^{13}$ had found increased resistance of SA and Streptococcus to ciprofloxacin and ofloxacin, with an overall resistance rate of 22 and 18\% to ciprofloxacin and ofloxacin, respectively, among the Gram-positive organisms, and 2.7\% among Gramnegative organisms for both antibiotics. Similar rate of resistance to ofloxacin (17\%) was found with the Gram positives in our study, but higher for the Gram negatives (10\%). Gram-negative organisms remained highly susceptible to ciprofloxacin $(98.9 \%)$ in our study but susceptibility was not tested in Gram-positive organisms as they are known to be highly resistant. ${ }^{13}$ We did not observe any emerging resistance to fluoroquinolones or any other commonly used antibiotics, which was also observed in other recent series. ${ }^{4,5,8}$ Conversely, there appeared to be an increasing susceptibility among the Gram-positive organisms to ofloxacin, specifically with the Streptococcus species, although the overall susceptibility rate of ofloxacin among the Streptococcus species was still low at $53.9 \%$. Decreasing antibiotic resistance or increasing susceptibility has been observed in other series. ${ }^{4,14}$ Taking into account our findings and recent literature, ofloxacin remains a reasonable first-line therapy in our centre but in cases that are slow to respond to treatment or when there is suspicion of Streptococcus species as a pathogen, switching to duo-therapy would be considered early. Our study suggested that combination therapy with vancomycin and ciprofloxacin covered 99.8 and $98.9 \%$ of Gram-positive and Gram-negative isolates, respectively, and these antibiotics could be considered in highly resistant cases especially where laboratory results are unavailable.

An important finding from this study was the observed trend change in the Gram-negative isolates. Pseudomonas species (mainly Pseudomonas aeruginosa) remained the commonest Gram-negative isolate over the study period (37.1\%), followed by Moraxella $(22.1 \%)$ but there was a sharp increase in the number of Moraxella species isolated in conjunction with what appeared to be the beginning of a trend towards declining Pseudomonas isolates towards the end of the study period (although this did not achieve statistical significance), despite an overall stable trend of the Gram-negative isolates. To the best of our knowledge, this trend has not been reported in recent literature. $P$. aeruginosa has been found to be the commonest pathogen associated with contact lens keratitis, 15,16 and the higher prevalence of these infections seen in Taiwan ${ }^{4}$ and China 9 appear to mirror the more frequent use of contact lenses. Other population studies ${ }^{8,10}$ have also observed a general decline in the incidence of Pseudomonas keratitis and this may be attributable to the increased awareness of contact lens hygiene. Moraxella species on the other hand, are a rarer causative pathogen for keratitis with a reported rate of $3.0-3.9 \%$ of all cultured proven bacterial keratitis. ${ }^{3,17}$ Inoue et al ${ }^{18}$ reviewed 30 cases of Moraxella keratitis retrospectively and found that contact lens wear was one of the predisposing factors for this infection but other ocular comorbidities including the use of steroid eye drops and poor ocular surface were also significant contributing factors. When patient age was more than 60 years, systemic comorbidities, such as diabetes, were an important predisposing factor. Other studies have also found Moraxella keratitis to be associated with chronic alcoholism, malnutrition, and poor sanitary habits. ${ }^{19-21}$ It is beyond the scope of this study to perform subgroup analysis on these two groups of patients. With the observed change in trend, high clinical suspicion for Moraxella keratitis is required especially in older patients with systemic comorbidities as the infection can appear clinically similar to Pseudomonas but may require a different antibiotic regime. According to our study, Moraxella species were $100 \%$ susceptible to chloramphenicol and cefuroxime and $99 \%$ to ciprofloxacin but only $88 \%$ susceptible to ofloxacin, as opposed to Pseudomonas species, which was known to be resistant to chloramphenicol ${ }^{22}$ but had high susceptibility to ciprofloxacin $(100 \%)$ and ofloxacin $(95 \%)$.

When considering non-bacterial isolates, $7.1 \%$ of our positive cultures grew fungi. The two most common fungal isolates reported in literature were yeast and Fusarium species, ${ }^{23,24}$ which was what we found in our study. Our study also observed a likely increasing trend of fungal infection, consistent with the findings from the US series. ${ }^{23,25}$ However, it is to be noted that the quoted studies reported fungal keratits in contact lens wearers specifically, unlike our study which reported the overall rate of fungal keratitis irrespective of aetiology. The firstline anti-fungal agent in our unit at the time of the study was voriconazole and our study confirmed a good susceptibility rate of $95.7 \%$ to the fungal species. In the mycotic ulcer treatment trial, ${ }^{26}$ natamycin has been recommended as the potential first-line therapy against filamentous fungi. Our laboratory does not routinely test for natamycin sensitivity and hence we are unable to comment on the use of this anti-fungal agent but voriconazole appeared to have a good anti-fungal activity overall in our study, for both Fusarium and yeast, in vitro at least. However, we note that recent study by Sharma et $a l^{27}$ confirmed a better clinical outcome with natamycin than voriconazole, especially in Fusarium keratitis and our centre is now considering switching to natamycin as the first line.

We found Acanthamoeba species in $2.3 \%$ of the positive cultures. This rate mirrors those of other studies. ${ }^{28,29}$ There appears to be a significant geographical variation, with some of the highest rates reported in the UK, where contact lens wear is more prevalent and the incidence is 
thought to be on the rise, which was also observed in our study. ${ }^{30-32}$ In our study, we found a higher incidence of fungal keratitis than Acanthamoeba keratitis. However, Acanthamoeba keratitis, some of which were culture negative and diagnosed based on clinical appearance and confocal microscopy, was undoubtedly seen and treated more commonly in our eye hospital over the study period than fungal keratitis, suggesting that corneal scrape in our institution might have a low sensitivity for Acanthamoeba. It is beyond the scope of this study to describe the incidence of culture-negative Acanthamoeba keratitis in our clinical practice but the actual rate of Acanthamoeba keratitis treated is expected to be higher than the rate reported here. The low incidence of culture-positive Acanthamoeba keratitis may be due to difficulty in culturing Acanthamoeba, whereas fungus grows more readily in cultures. While culture remains the gold standard for diagnosing Acanthamoeba, several polymerase chain reaction-based techniques have been described to increase sensitivity significantly. ${ }^{33,34}$

There are a few limitations in this study. There is a possibility that the positive cultures in some of the cases were caused by non-pathogenic commensals from the ocular surface, such as the CNS. The converse is also true where the pathogens may not have been cultured successfully due to inadequate corneal sample or limitations in laboratory techniques. Therefore, the decreasing trend of Gram-positive organisms needs to be interpreted with care as it may not be representative of the actual trends of disease-causing pathogens. Like other antibiotics susceptibility studies, in vitro testing of susceptibility and resistance does not always translate into real-life clinical response as frequency of use, host factors, and penetration of eye drops may all have a role in the efficacy of an antibiotic. Another limitation in this study includes the selective testing of antibiotics against certain isolates based on prior knowledge of susceptibility. This may have introduced bias in the rates reported and any conclusions drawn from this study need to take this limitation in methodology into account. We were also unable to investigate the change in trend in susceptibility, if any, of neither the commonly used antibiotics against all the isolates nor the newer antibiotics, such as moxifloxacin, gatifloxacin, or levofloxacin, due to the retrospective nature of this study. As there is geographical difference in the pathogenicity of microorganisms, care has to be taken when extrapolating the results from our study to other regions.

In conclusion, we found a change in the type of Gram-negative organisms isolated, with the Moraxella species on the rise. It is reassuring that there was no observed increase in resistance in any of the commonly used antibiotics tested in vitro. Ofloxacin remains a good first-line antibiotic treatment but duo-therapy should be considered early in clinically non-responsive cases.

Summary

What was known before

- Pseudomonas is the commonest Gram-negative organism in microbial keratitis in many studies. There have been concerns about emerging resistance to commonly used antibiotics.

What this study adds

- Moraxella keratitis is on the rise. No increase in resistance was observed for any of the commonly used antibiotics such as ofloxacin.

\section{Conflict of interest}

The authors declare no conflict of interest.

\section{References}

1 Green MD, Apel AJ, Naduvilath T, Stapleton FJ. Clinical outcomes of keratitis. Clin Exp Ophthalmol 2007; 35(5): 421-426.

2 Five Year UK. Antimicrobial resistance strategy 2013 to 2018. Department of Health. Available at: http://www.gov.uk/ government/uploads/system/uploads/attachment_data/ file/244058/20130902_UK_5_year_AMR_strategy.pdf.

3 Orlans HO, Hornby SJ, Bowler IC. In vitro antibiotic susceptibility patterns of bacterial keratitis isolates in Oxford, UK: a 10-year review. Eye 2011; 25(4): 489-493.

4 Hsiao CH, Sun CC, Yeh LK, Ma DH, Chen PY, Lin HC et al. Shifting trends in bacterial keratitis in Taiwan: a 10-year review in a tertiary-care hospital. Cornea 2016; 35(3): 313-317.

5 Ferreira CS, Figueira L, Moreira-Gonçalves N, Moreira R, Torrão L, Falcão-Reis F. Clinical and microbiological profile of bacterial microbial keratitis in a portuguese tertiary referral center-where are we in 2015? Eye Contact Lens 2016; e-pub ahead of print 12 July 2016; doi:10.1097/ICL.0000000 000000298.

6 Kaye S, Tuft S, Neal T, Tole D, Leeming J, Figueiredo F et al. Bacterial susceptibility to topical antimicrobials and clinical outcome in bacterial keratitis. Invest Ophthalmol Vis Sci 2010; 51(1): 362-368.

7 Gopinathan U, Sharma S, Garg P, Rao GN. Review of epidemiological features, microbiological diagnosis and treatment outcome of microbial keratitis: experience of over a decade. Indian J Ophthalmol 2009; 57(4): 273-279.

8 Al-Dhaheri HS, Al-Tamimi MD, Khandekar RB, Khan M, Stone DU. Ocular pathogens and antibiotic sensitivity in bacterial keratitis isolates at King Khaled Eye Specialist Hospital, 2011 to 2014. Cornea 2016; 35(6): 789-794.

9 Zhang C, Liang Y, Deng S, Wang Z, Li R, Sun X. Distribution of bacterial keratitis and emerging resistance to antibiotics in China from 2001 to 2004. Clin Ophthalmol 2008; 2(3): 575-579.

10 Alexandrakis G, Alfonso EC, Miller D. Shifting trends in bacterial keratitis in south Florida and emerging resistance to fluoroquinolones. Ophthalmology 2000; 107(8): 1497-1502. 
11 Wilhelmus KR, Abshire RL, Schlech BA. Influence of fluoroquinolone susceptibility on the therapeutic response of fluoroquinolone-treated bacterial keratitis. Arch Ophthalmol 2003; 121(9): 1229-1233.

12 O'Brien TP, Maguire MG, Fink NE, Alfonso E, McDonnell P. Efficacy of ofloxacin vs cefazolin and tobramycin in the therapy for bacterial keratitis. Report from the Bacterial Keratitis Study Research Group. Arch Ophthalmol 1995; 113(10): 1257-1265.

13 Goldstein MH, Kowalski RP, Gordon YJ. Emerging fluoroquinolone resistance in bacterial keratitis: a 5-year review. Ophthalmology 1999; 106(7): 1313-1318.

14 Chalita MR, Höfling-Lima AL, Paranhos A, Schor P, Belfort R. Shifting trends in in vitro antibiotic susceptibilities for common ocular isolates during a period of 15 years. Am J Ophthalmol 2004; 137(1): 43-51.

15 Hoddenbach JG, Boekhoorn SS, Wubbels R, Vreugdenhil W, Van Rooij J, Geerards AJ. Clinical presentation and morbidity of contact lens-associated microbial keratitis: a retrospective study. Graefes Arch Clin Exp Ophthalmol 2014; 252(2): 299-306.

16 Konda N, Motukupally SR, Garg P, Sharma S, Ali MH, Willcox MD. Microbial analyses of contact lens-associated microbial keratitis. Optom Vis Sci 2014; 91(1): 47-53.

17 Japan NSoIKi. National surveillance of infectious keratitis in Japan-current status of isolates, patient background, and treatment. Nippon Ganka Gakkai Zasshi 2006; 110(12): 961-972.

18 Inoue $\mathrm{H}$, Suzuki T, Inoue T, Hattori T, Nejima R, Todokoro D et al. Clinical characteristics and bacteriological profile of Moraxella keratitis. Cornea 2015; 34(9): 1105-1109.

19 Heidemann DG, Alfonso E, Forster RK, Ullman S, Holland SP, Mendelsohn A et al. Branhamella catarrhalis keratitis. Am J Ophthalmol 1987; 103(4): 576-581.

20 Marioneaux SJ, Cohen EJ, Arentsen JJ, Laibson PR. Moraxella keratitis. Cornea 1991; 10(1): 21-24.

21 Baum J, Fedukowicz HB, Jordan A. A survey of Moraxella corneal ulcers in a derelict population. Am J Ophthalmol 1980; 90(4): 476-480.

22 Bourkiza R, Kaye S, Bunce C, Shankar J, Neal T, Tuft S. Initial treatment of Pseudomonas aeruginosa contact lens-associated keratitis with topical chloramphenicol, and effect on outcome. Br J Ophthalmol 2013; 97(4): 429-432.
23 Jurkunas U, Behlau I, Colby K. Fungal keratitis: changing pathogens and risk factors. Cornea 2009; 28(6): 638-643.

24 Iyer SA, Tuli SS, Wagoner RC. Fungal keratitis: emerging trends and treatment outcomes. Eye Contact Lens 2006; 32(6): 267-271.

25 Yildiz EH, Abdalla YF, Elsahn AF, Rapuano CJ, Hammersmith KM, Laibson PR et al. Update on fungal keratitis from 1999 to 2008. Cornea 2010; 29(12): 1406-1411.

26 Sun CQ, Lalitha P, Prajna NV, Karpagam R, Geetha M, $\mathrm{O}^{\prime}$ Brien KS et al. Association between in vitro susceptibility to natamycin and voriconazole and clinical outcomes in fungal keratitis. Ophthalmology 2014; 121(8): 1495-1500. e1491.

27 Sharma S, Das S, Virdi A, Fernandes M, Sahu SK, Kumar Koday N et al. Re-appraisal of topical $1 \%$ voriconazole and $5 \%$ natamycin in the treatment of fungal keratitis in a randomised trial. Br J Ophthalmol 2015; 99(9): 1190-1195.

28 Houang E, Lam D, Fan D, Seal D. Microbial keratitis in Hong Kong: relationship to climate, environment and contact-lens disinfection. Trans R Soc Trop Med Hyg 2001; 95(4): 361-367.

29 Butler TK, Males JJ, Robinson LP, Wechsler AW, Sutton GL, Cheng J et al. Six-year review of Acanthamoeba keratitis in New South Wales, Australia: 1997-2002. Clin Exp Ophthalmol 2005; 33(1): 41-46.

30 Dart JK, Saw VP, Kilvington S. Acanthamoeba keratitis: diagnosis and treatment update 2009. Am J Ophthalmol 2009; 148(4): 487-499.e482.

31 Srinivasan M, Gonzales CA, George C, Cevallos V, Mascarenhas JM, Asokan B et al. Epidemiology and aetiological diagnosis of corneal ulceration in Madurai, south India. Br J Ophthalmol 1997; 81(11): 965-971.

32 Maycock NJ, Jayaswal R. Update on Acanthamoeba keratitis: diagnosis, treatment, and outcomes. Cornea 2016; 35(5): 713-720.

33 Ikeda Y, Miyazaki D, Yakura K, Kawaguchi A, Ishikura R, Inoue $\mathrm{Y}$ et al. Assessment of real-time polymerase chain reaction detection of Acanthamoeba and prognosis determinants of Acanthamoeba keratitis. Ophthalmology 2012; 119(6): 1111-1119.

34 Mathers WD, Nelson SE, Lane JL, Wilson ME, Allen RC, Folberg R. Confirmation of confocal microscopy diagnosis of Acanthamoeba keratitis using polymerase chain reaction analysis. Arch Ophthalmol 2000; 118(2): 178-183. 